$\operatorname{gr}-\mathrm{qc} / \ldots$

\title{
Stability in Designer Gravity
}

\author{
Thomas Hertog ${ }^{1}$ and Stefan Hollands ${ }^{1,2}$ \\ ${ }^{1}$ Department of Physics, UCSB, Santa Barbara, CA 93106 \\ ${ }^{2}$ Inst. f. Theor. Physik, Georg-August-Universität, D-37077 Göttingen
}

\begin{abstract}
We study the stability of designer gravity theories, in which one considers gravity coupled to a tachyonic scalar with anti-de Sitter boundary conditions defined by a smooth function $W$. We construct Hamiltonian generators of the asymptotic symmetries using the covariant phase space method of Wald et al. and find they differ from the spinor charges except when $W=0$. The positivity of the spinor charge is used to establish a lower bound on the conserved energy of any solution that satisfies boundary conditions for which $W$ has a global minimum. A large class of designer gravity theories therefore have a stable ground state, which the AdS/CFT correspondence indicates should be the lowest energy soliton. We make progress towards proving this, by showing that minimum energy solutions are static. The generalization of our results to designer gravity theories in higher dimensions involving several tachyonic scalars is discussed.
\end{abstract}

\footnotetext{
${ }^{1}$ hertog@vulcan.physics.ucsb.edu

${ }^{2}$ hollands@theorie.physik.uni-goettingen.de
} 


\section{Introduction}

In theories of gravity coupled to matter, the theory is usually fully determined by the action. The boundary conditions at infinity are often not independent, but uniquely determined by basic requirements such as finite total energy. This is not the case, however, for gravity coupled to certain scalar field theories in asymptotically anti-de Sitter (AdS) spacetimes. In particular, it has been shown that scalars $\phi$ with mass $m^{2}$ at or slightly above the Breitenlohner-Freedman (BF) bound [1] admit a large class of boundary conditions, defined by an essentially arbitrary real function $W$, which prescribes the relation between the asymptotic value of the scalar field and its normal derivative at infinity. Furthermore, for each choice of $W$, one can derive manifestly finite expressions for the total energy and angular momentum of the theory [2].

Theories of this type were called designer gravity theories, since their properties depend significantly on the choice of boundary conditions. By choosing the appropriate boundary condition function $W$ one can, for example, specify the number as well as the masses of spherical soliton solutions. It was also found that certain boundary conditions admit AdS-black holes with scalar hair [3, 4, 5]. Designer gravity theories therefore provide a useful context in which one can study what are the requirements on scalar matter for the no hair theorems to hold.

Interesting examples of designer gravity theories arise in string theory compactifications on a sphere. String theory with $A d S_{4} \times S^{7}$ boundary conditions, for instance, reduces at low energies to $\mathcal{N}=8$ gauged supergravity in four dimensions, which contains several $m^{2}=-2$ scalars. It is an obvious question which - if any-choices of $W$ would yield a stable theory, i.e., one whose energy is bounded from below. Furthermore, in such a theory, it would be of interest to classify all solutions that locally minimize the energy.

Insight into these questions can be gained from the AdS/CFT correspondence [6]. This states that, with $W=0$ boundary conditions, the gravity theory is equivalent to a $2+1$ conformal field theory (CFT) that is defined on the boundary spacetime. Adopting more general $W \neq 0$ boundary conditions on one (or several) of the tachyonic scalars corresponds to the addition of a potential term $\int W(\mathcal{O})$ to the dual CFT action, where $\mathcal{O}$ is the field theory operator that is dual to the bulk scalar $[7,8]$. Based on the AdS/CFT duality, it was conjectured in [2] that (a) there is a lower bound 
on the gravitational energy in those designer gravity theories where $W$ is bounded from below and that (b) the solutions locally minimizing the energy are given by the spherically symmetric, static soliton configurations found in [2].

In this paper, we make progress towards proving these conjectures. First, we prove that if the function $W$ is bounded from below, then so is the energy of the gravity theory. In fact, we present a lower bound on the gravitational energy in terms of the global minumum of $W$, see eq. (3.11). Second, we prove that, under certain technical assumptions, local minima of the energy must be static, i.e., admit a globally defined surface orthogonal timelike Killing field. However, we have not yet been able to fully characterize these minima. In particular, we do not know whether they are given by the spherical soliton solutions found in [2], or whether they encompass a wider class of solutions. Progress towards these problems can perhaps be made most easily by numerical methods.

Our proofs of these statements only involve arguments in the classical gravity theory, and do not rely on the AdS/CFT correspondence. However, the lower bound (3.11) agrees with the lower bound on the energy of dual field theory configurations. Our result can therefore be viewed as a test of the AdS/CFT correspondence.

We also outline the generalization of our results to other designer gravity theories in higher dimensions involving several tachyonic scalar fields.

Our notations and conventions are the same as in [9]. In particular, indices on tilded tensor fields $\tilde{t}_{a b c . .}$ are raised and lowered with the unphysical metric $\tilde{g}_{a b}$ and its inverse, whereas indices on untilded fields are raised and lowered with the physical metric $g_{a b}=\Omega^{-2} \tilde{g}_{a b}$, and its inverse. We set the AdS radius and $8 \pi G$ equal to one.

\section{Asymptotics and Conserved Charges}

Most generally, we are interested in theories of gravity coupled to one or more scalar fields with potential $V(\phi)$. In particular, we will consider potentials that have a critical point $\phi_{0}$ where $V\left(\phi_{0}\right)<0$ and where certain small scalar fluctuations are tachyonic, $m^{2}<0$, with the scalar mass in the range

$$
m_{B F}^{2} \leq m^{2}<m_{B F}^{2}+1
$$


Here $m_{B F}^{2}=-(d-1)^{2} / 4$ is the Breitenlohner-Freedman (BF) bound [1] in $d$ spacetime dimensions. For definiteness we will focus on the case of $\mathcal{N}=8$ gauged supergravity in $d=4$ dimensions and comment on generalizations at the end. This theory can be consistently truncated to include just gravity and a single scalar field [10], given by the Lagrangian density (viewed as a 4 -form)

$$
\mathbf{L}=\frac{1}{2} d^{4} x \sqrt{-g}\left[R-(\nabla \phi)^{2}-2 V(\phi)\right]
$$

with

$$
V(\phi)=-2-\cosh \sqrt{2} \phi
$$

The potential (2.3) has a maximum at $\phi=0$. It is unbounded from below, but small fluctuations have $m^{2}=-2$, which is above the BF bound and within the range (2.1). To specify the theory completely, we must also provide a set of boundary conditions, to which we now turn.

\subsection{Asymptotic Conditions}

1. One can attach a boundary, $\mathcal{I} \cong \mathbb{R} \times S^{2}$ to $M$ such that $\tilde{M}=M \cup \mathcal{I}$ is a manifold with boundary.

2. On $\tilde{M}$, there is a $\operatorname{smooth}^{3}$ function $\Omega$, with $\Omega=0$ and $\tilde{n}_{a}=\tilde{\nabla}_{a} \Omega \neq 0$ on $\mathcal{I}$, such that

$$
\tilde{g}_{a b}=\Omega^{2} g_{a b}, \quad \tilde{\phi}=\Omega^{-1} \phi
$$

are smooth at $\mathcal{I}$. We also require that

$$
d \widetilde{s}^{2}=d \Omega^{2}-d t^{2}+d \theta^{2}+\sin ^{2} \theta d \varphi^{2}+\ldots \quad \text { near } \mathcal{I}
$$

and

$$
\tilde{\phi}=\alpha+\beta \Omega+\ldots \quad \text { near } \mathcal{I}
$$

Here, $\alpha$ is a smooth function on $\mathcal{I}$. We further assume there is a functional relation between $\alpha$ and $\beta$,

$$
\beta=W^{\prime}(\alpha)
$$

where $W$ is a smooth function of $\alpha$ with $W(0)=W^{\prime}(0)=0$.

\footnotetext{
${ }^{3}$ By "smooth", we always mean $C^{\infty}$.
} 
The prototype spacetime satisfying these asymptotic conditions (for any $W$ ) is pure AdS space with $\phi=0$ everywhere. In global coordinates its metric is given by

$$
d s_{0}^{2}=-\left(1+r^{2}\right) d t^{2}+\frac{d r^{2}}{1+r^{2}}+r^{2}\left(d \theta^{2}+\sin ^{2} \theta d \varphi^{2}\right),
$$

which can be brought into the form (2.4)-(2.5) e.g. by choosing $\Omega=r^{-1}$. Thus, for pure AdS, a conformal completion can be obtained by taking $\tilde{M}$ to be the manifold obtained from $M$ by attaching the boundary $\mathcal{I}$ consisting of the points $\Omega=0$, and by taking the unphysical metric to be $d \tilde{s}_{0}^{2}=\Omega^{2} d s_{0}^{2}$. One can likewise verify that the asymptotic conditions are also obeyed by the AdS-Schwarzschild and the AdS-MyersPerry solutions, in which the scalar field vanishes.

If the above asymptotic conditions are combined with Einstein's equations, then one can obtain much more information on the asymptotic form of the metric near infinity. For instance, by using the expansion techniques of [11] one finds

$$
\begin{aligned}
d \tilde{s}^{2} & =d \Omega^{2}-\left(1+\frac{2-\alpha^{2}}{4} \Omega^{2}-\frac{4 \alpha \beta}{9} \Omega^{3}\right) d t^{2} \\
& +\left(1-\frac{2+\alpha^{2}}{4} \Omega^{2}-\frac{4 \alpha \beta}{9} \Omega^{3}\right) d \sigma^{2} \\
& -\frac{2}{3} \Omega^{3} \mathcal{E}_{a b} d x^{a} d x^{b}+\ldots,
\end{aligned}
$$

where $d \sigma^{2}=d \theta^{2}+\sin ^{2} \theta d \varphi^{2}$. The asymptotic expansion of the physical line element $d s^{2}$ is obtained by dividing the above expressions by $\Omega^{2}$. The quantity $\mathcal{E}_{a b}$ is the leading order electric Weyl tensor, defined by

$$
\mathcal{E}_{a b}=\Omega^{-1} \tilde{C}_{a c b d} \tilde{n}^{c} \tilde{n}^{d} .
$$

This can be shown to be smooth at $\mathcal{I}$ if the field equations are satisfied, despite the inverse power of $\Omega$.

It is well known that with 'reflective' boundary conditions, defined by $\alpha=0$, tachyonic scalars in AdS spacetime do not cause an instability provided their mass is above the BF bound. With $\alpha=0$ boundary conditions our theory (2.2) admits a positive energy theorem $[22,23]$, which ensures the total energy cannot be negative whenever this condition is satisfied.

In this paper we are concerned with a different class of boundary conditions, which we defined above by a function $W(\alpha)$. Although these boundary conditions are not 
in general invareiant under the full AdS symmetry group, they are invariant under global time translations so one can still define a conserved energy. In $[3,12,2]$ the conserved energy was constructed within the Hamiltonian framework of Henneaux and Teitelboim [13]. It was found that for all $W$ there is an additional surface term associated with the scalar field, which renders the conserved energy finite. Here we study the stability of theories such as (2.2), with boundary conditions (2.5)-(2.7). In particular, we wish to prove the conjecture of [2], which states that the energy is bounded from below whenever $W$ has a global minimum.

But first we present an alternative construction of the conserved charges in this context, in which we adopt the covariant phase space formalism of Wald et al. [14]. This provides a general algorithm for defining charges associated with symmetries preserving a given set of boundary conditions, and it will be useful when we come to analyze the positivity properties of the charges.

\subsection{Conserved Charges}

Asymptotic symmetries are diffeomorphisms $f$ of $\tilde{M}$ that preserve a prescribed set of boundary conditions. That is, if a solution $\Phi=\left(\phi, g_{a b}\right)$ satisfies a given set of boundary conditions, then $f^{*} \Phi$ does, too. The asymptotic symmetries form an infinitedimensional group. Of physical interest is the factor group $G=\operatorname{Diff}(M) / \operatorname{Diff}(M)_{0}$, where $\operatorname{Diff}(M)_{0}$ is the subgroup of diffeos leaving a neighborhood of $\mathcal{I}$ pointwise invariant. For designer gravity boundary conditions defined by a generic $W$, the elements of $G$ can be identified with isometries of the Einstein static universe. When $W(\alpha) \propto \alpha^{3}$, however, $G$ is larger and corresponds to the conformal isometry group of the Einstein static universe, $G \cong O(3,2)[3,12]$. The Lie algebra of $G$ consists of vector fields $\xi^{a}$ tangent to $\mathcal{I}$ that Lie derive the metric of the Einstein static universe, modulo vector fields that vanish on $\mathcal{I}$. For simplicity, we will also refer to such vector fields as asymptotic symmetries. We now carefully define the generators $\mathcal{H}_{\xi}$ on phase space associated with the asymptotic symmetries that leave invariant the above set of boundary conditions ${ }^{4}$.

\footnotetext{
${ }^{4}$ Note that, since an infinitesimal asymptotic symmetry is only specified modulo vector fields on $\tilde{M}$ that vanish on $\mathcal{I}$, it follows that $\mathcal{H}_{\xi}$ can only depend on this equivalence class, i.e., it must vanish for any vector field that is zero on $\mathcal{I}$. This means, roughly speaking, that $\mathcal{H}_{\xi}$ cannot depend on derivatives of $\xi$ normal to $\mathcal{I}$.
} 
Consider first the variation of the Lagrange density $\mathbf{L}$. This can always be written in the form

$$
\delta \mathbf{L}=\mathbf{F} \cdot \delta \Phi+d \boldsymbol{\theta},
$$

Here, $\delta \Phi=\left(\delta g_{a b}, \delta \phi\right)$, the symbol $\mathbf{F}$ represents the field equations and $d \boldsymbol{\theta}$ is the exterior differential of the 3-form $\boldsymbol{\theta}$ that corresponds to the boundary term which would arise if the variation of $\mathbf{L}$ were performed under an integral sign. For the Lagrangian (2.2) it is given by

$$
\theta_{a b c}=\frac{1}{2}\left(\nabla^{d} \delta g_{e}^{e}-\nabla^{e} \delta g_{e}{ }^{d}+2 \delta \phi \nabla^{d} \phi\right) \epsilon_{a b c d},
$$

where $\boldsymbol{\epsilon}=d^{4} x \sqrt{-g}$ is the volume form (identified with a 4 -form). The antisymmetrized second variation $\boldsymbol{\omega}$ of $\boldsymbol{\theta}$ defines the (dualized) symplectic current,

$$
\boldsymbol{\omega}\left(\Phi ; \delta_{1} \Phi, \delta_{2} \Phi\right)=\delta_{1} \boldsymbol{\theta}\left(\Phi ; \delta_{2} \Phi\right)-\delta_{2} \boldsymbol{\theta}\left(\Phi ; \delta_{1} \Phi\right)
$$

so that $\boldsymbol{\omega}$ depends on the unperturbed metric and scalar field and is skew in the pair of perturbations.

The integral of the symplectic current over an achronal 3-dimensional submanifold $\Sigma$ defines the symplectic structure, $\sigma_{\Sigma}$, of the theory

$$
\sigma_{\Sigma}\left(\Phi, \delta_{1} \Phi, \delta_{2} \Phi\right)=\int_{\Sigma} \boldsymbol{\omega}\left(\Phi ; \delta_{1} \Phi, \delta_{2} \Phi\right) .
$$

It follows from a general argument that if both perturbations satisfy the linearized equations of motion, then the symplectic current is conserved, $d \boldsymbol{\omega}=0$. From this one can deduce how $\sigma_{\Sigma}$ depends upon the choice of $\Sigma$. This goes as follows. Let $\Sigma_{1}$ and $\Sigma_{2}$ two achronal surfaces ending on $\mathcal{I}$, which enclose a spacetime volume that is bounded by $\Sigma_{1}, \Sigma_{2}$, and the portion $\mathcal{I}_{12}$ of infinity bounded by the corresponding cuts $C_{1}$ and $C_{2}$ on which the surfaces end. By Stokes theorem, the difference between the symplectic form on both 3 -surfaces is given by an integral of $d \boldsymbol{\omega}$ over the spacetime volume, plus the integral $\int_{\mathcal{I}_{12}} \boldsymbol{\omega}$. However, for perturbations satisfying the linearized equations of motion, the symplectic current is given by

$$
\begin{aligned}
\omega_{a b c}\lceil\mathcal{I} & =\left(\delta_{1} \beta \delta_{2} \alpha-\delta_{2} \beta \delta_{1} \alpha\right) \tilde{\epsilon}_{a b c} \\
& =W^{\prime \prime}(\alpha)\left(\delta_{1} \alpha \delta_{2} \alpha-\delta_{2} \alpha \delta_{1} \alpha\right) \tilde{\epsilon}_{a b c}=0
\end{aligned}
$$


on $\mathcal{I}$, where $\tilde{\epsilon}_{a b c}$ is the integration element on $\mathcal{I}$ induced by $\tilde{g}_{a b}$, i.e., $4 \tilde{n}_{[a} \tilde{\epsilon}_{b c d]}=\tilde{\epsilon}_{a b c d}$. Hence the symplectic structure $\sigma_{\Sigma}$ is independent of the choice of $\Sigma$. Note that this would not have been the case if $\alpha$ and $\beta$ had been two independent functions.

One would like to define the generator associated with a vector field $\xi^{a}$ representing an asymptotic symmetry by

$$
\delta \mathcal{H}_{\xi}=\sigma_{\Sigma}\left(\Phi ; \delta \Phi, £_{\xi} \Phi\right) \quad \forall \delta \Phi
$$

where $\Sigma$ is a partial Cauchy surface whose boundary, $S_{\infty}^{2}$, is a cut of $\mathcal{I}$. This definition would imply that $\mathcal{H}_{\xi}$-if it exists - generates (in the sense of Hamiltonian mechanics) the infinitesimal displacement ("Hamiltonian vector field") $\delta \Phi=£_{\xi} \Phi$, which in turn describes the action of an infinitesimal symmetry in the phase space of the theory. In addition, $\mathcal{H}_{\xi}$ would not depend on the cut $S_{\infty}^{2}$ chosen, since the symplectic form is independent of the choice of $\Sigma$. Hence, if one can indeed construct $\mathcal{H}_{\xi}$ in this way, it will be automatically conserved. Moreover, it also follows that the Hamiltonian vector fields associated with two infinitesimal symmetries $\xi^{a}, \eta^{a}$ satisfy the same algebra as ordinary vector fields on $M$ under the commutator [11].

To analyze the existence of $\mathcal{H}_{\xi}$ it is instructive, following [14], to first study the general structure of eq. (2.16). The right hand side of (2.16) can be written as [14]

$$
\delta \mathcal{H}_{\xi}=\int_{\Sigma} \delta \mathbf{C}_{a} \xi^{a}+\int_{S_{\infty}^{2}}\left[\delta \mathbf{Q}_{\xi}-\xi \cdot \boldsymbol{\theta}\right]
$$

Here, $\mathbf{C}_{a}$ are the constraints of the theory (identified with 3 -forms), and $\mathbf{Q}_{\xi}$ is the Noether charge, which is given in our case by

$$
Q_{a b}=-\frac{1}{2}\left(\nabla^{c} \xi^{d}\right) \epsilon_{a b c d} .
$$

Consistency requires $\left(\delta_{1} \delta_{2}-\delta_{2} \delta_{1}\right) \mathcal{H}_{\xi}=0$, so we must have

$$
0=\xi \cdot\left[\delta_{2} \boldsymbol{\theta}\left(\Phi, \delta_{1} \Phi\right)-\delta_{1} \boldsymbol{\theta}\left(\Phi, \delta_{2} \Phi\right)\right]=\xi \cdot \boldsymbol{\omega}\left(\Phi ; \delta_{1} \Phi, \delta_{2} \Phi\right)
$$

on $\mathcal{I}$, or else $\mathcal{H}_{\xi}$ cannot exist. But this follows immediately from (2.15). Here it enters there is a functional relation between $\beta$ and $\alpha$, illustrating clearly that a complete set of boundary conditions includes a specification of $W$.

It follows from the consistency condition that there is a 2 -form $\mathbf{I}_{\xi}$ such that

$$
\delta \mathbf{Q}_{\xi}-\xi \cdot \boldsymbol{\theta}=\delta \mathbf{I}_{\xi}
$$


up to an exact form. We conclude that a solution to (2.17) exists and is given by

$$
\mathcal{H}_{\xi}=\int_{\Sigma} \xi^{a} \mathbf{C}_{a}+\int_{S_{\infty}^{2}} \mathbf{I}_{\xi}
$$

If the equations of motion are satisfied then the constraints $\mathbf{C}_{a}$ vanish identically and $\mathcal{H}_{\xi}$ reduces to a surface integral. Finally, from (2.9), (2.12) and (2.18) it follows that a generator $\mathcal{H}_{\xi}$ that satisfies $(2.21)$ is given explicitly by ${ }^{5}$

$$
\mathcal{H}_{\xi}=-\int_{S_{\infty}^{2}} \mathcal{E}_{a b} t^{a} \xi^{b} \sqrt{\sigma} d^{2} x-\int_{S_{\infty}^{2}}\left[W(\alpha)-\frac{1}{3} \alpha \beta\right] t^{a} \xi_{a} \sqrt{\sigma} d^{2} x,
$$

where the cut at infinity has been chosen, without loss of generality, as the $S^{2}$ orthogonal to $t^{a}=(\partial / \partial t)^{a}$. Since $\mathcal{H}_{\xi}$ is defined through its variation, one can always add an arbitrary constant to (2.22). This constant is usually determined by requiring that $\mathcal{H}_{\xi}$ vanishes on exact $\mathrm{AdS}$ space with $\phi=0$ everywhere, which holds for the above expression provided $W(0)=0$.

When $\xi^{a}=(\partial / \partial t)^{a}, \mathcal{H}_{\partial / \partial t}$ is the energy $E$ of the field configuration $\left(g_{a b}, \phi\right)$. The energy has a contribution from the electric Weyl tensor of the gravitational field, and an extra term that depends on the asymptotic profile of the scalar field and on the choice of boundary conditions defined by the function $W$. Note that the contribution from the scalar is absent for the generalized conformally invariant boundary conditions $\beta \propto \alpha^{2}$, as well as for the "standard" boundary conditions $\alpha=0$. When $\xi^{a}=(\partial / \partial \varphi)^{a}$ is a rotational Killing field on $S^{2}$, then $\mathcal{H}_{\partial / \partial \varphi}$ is the angular momentum $J$, and the expression for $\mathcal{H}_{\partial / \partial \varphi}$ coincides with the one obtained with standard boundary conditions.

The charges (2.22), which we defined by using the covariant phase space formalism of Wald et al. [14], agree with the charges one obtains using the Henneaux-Teitelboim formalism [13]. Indeed, with generalized boundary conditions (2.6) one finds the latter also acquire an explicit scalar contribution [3, 12]. On the other hand, the definition of Ashtekar et al. [15], which is based purely on the electric Weyl tensor, obviously yields different charges in this context, which are in general not conserved. Finally, we expect that it should also be possible to construct the charges in designer gravity using the "counterterm subtraction method" [16, 17]. Based on the general arguments

\footnotetext{
${ }^{5}$ We thank Aaron Amsel for performing an independent check of (2.22), and for filling in a gap in our original calculation.
} 
presented in [18], we expect that the counterterm approach should yield charges that are equal to our charges (2.22), up to a constant offset.

In the next section we compare our charges with the spinor charges [23, 24], and use this relationship to analyze the positivity properties of the energy.

\section{Lower Bound on the Energy}

The conserved energy $E=\mathcal{H}_{\partial / \partial t}$ cannot satisfy a positive energy theorem for all choices of boundary conditions, since for certain $W(\alpha)$ there exist globally smooth solutions with negative energy. Indeed, in [5] it was shown that our theory (2.2) with $\beta=-k \alpha^{2}+\epsilon \alpha^{3}$ boundary conditions (with $k \gg \epsilon>0$ ) admits e.g. negative energy spherical solitons. Furthermore, with AdS-invariant boundary conditions $\beta=-k \alpha^{2}$ one can even construct smooth solutions that have arbitrary negative energy [19]. This raises the question what (if any) choices of boundary conditions $W$ in designer gravity yield a theory with a stable ground state. In this section we address this point. In particular, we show there is a lower bound on the energy $E$ of any solution that satisfies boundary conditions for which $W$ has a global minimum. The central idea of our proof is to relate the Hamiltonian charges (2.22) to the spinor charges, which can be shown to be manifestly positive for all $W$.

First consider a future directed timelike vector field $\xi^{a}$ for which asymptotically

$$
\xi^{a}=\left(\frac{\partial}{\partial t}\right)^{a}+\omega\left(\frac{\partial}{\partial \varphi}\right)^{a}, \quad \text { on } \mathcal{I}
$$

where $-1<\omega<1$ is a real constant. Assume that $M$ admits a spin structure, and let $\psi$ be a spinor field on $M$ such that asymptotically $\bar{\psi} \gamma^{a} \psi=\xi^{a}$. Let $\mathbf{B}$ be the 2 -form with components

$$
B_{a b}=\frac{1}{2}\left(\bar{\psi} \gamma^{[c} \gamma^{d} \gamma^{e]} \widehat{\nabla}_{e} \psi+\text { h.c. }\right) \epsilon_{a b c d},
$$

where $\gamma_{a}$ are the curved space gamma matrices and

$$
\widehat{\nabla}_{a} \psi=\left[\nabla_{a}-\frac{1}{2} \gamma_{a} \cosh (\phi / \sqrt{2})\right] \psi .
$$

The spinor charge is defined by

$$
\mathcal{Q}_{\xi}=\int_{S_{\infty}^{2}} \mathbf{B}
$$


The following identity [24] allows one to prove its positivity:

$$
(d \mathbf{B})_{i j k}=\left[2\left(\widehat{D}_{l} \psi, \widehat{D}^{l} \psi\right)+|\lambda|^{2}\right] \epsilon_{i j k}-2\left|\gamma^{l} \widehat{D}_{l} \psi\right|^{2} \epsilon_{i j k}
$$

with

$$
\lambda=\frac{1}{\sqrt{2}} \gamma^{a}\left(\nabla_{a} \phi\right) \psi+\sinh (\phi / \sqrt{2}) \psi
$$

Here $i, j, \ldots$ denote indices tangent to a spacelike 3 -surface $\Sigma$ spanning the $S_{\infty}^{2}$ at infinity, $\epsilon_{i j k}$ is the induced volume 3 -form, and $\widehat{D}_{i}$ is the derivative operator obtained from $\widehat{\nabla}_{a}$ by projecting the tangent space index into $\Sigma$. Finally, the hermitian inner product $(\lambda, \lambda)$ is defined by $\bar{\lambda} \gamma^{a} \lambda u_{a}$, where $u^{a}$ is the unit timelike normal to $\Sigma$. The first term in $d \mathbf{B}$ is a manifestly non-negative density. The second term can be negative, but it can be set to zero by requiring $\psi$ to satisfy the Witten condition,

$$
\gamma^{i} \widehat{D}_{i} \psi=0
$$

In Appendix A we prove that our choice of boundary conditions indeed allows one to impose this condition, and at the same time require that asymptotically $\bar{\psi} \gamma^{a} \psi=$ $\xi^{a}$. Assuming that $\Sigma$ has no boundary components other than $S_{\infty}^{2}$ (such as inner boundaries ending on the horizon of a black hole), it now follows from Stokes theorem that $\mathcal{Q}_{\xi}=\int_{\Sigma} d \mathbf{B} \geq 0$.

Now define $\tilde{\psi}=\Omega^{1 / 2} \psi$ and $\tilde{\gamma}_{a}=\Omega \gamma_{a}$, which are smooth functions on the boundary $\mathcal{I}$, and write the 2 -form $B_{a b}$ as $\Omega^{-2} \tilde{B}_{a b}$. The 2 -form $\tilde{B}_{a b}$ is given by the same expression (3.2), but with all quantities replaced by their corresponding tilded quantities and with $\widehat{\nabla}_{a}$ replaced by $\Omega^{1 / 2} \widehat{\nabla}_{a} \Omega^{-1 / 2}$. From the asymptotic expansion (A.5) of the spinor field $\psi$, and the asymptotic expansions of the metric and the scalar field $\phi$, eqs. (2.9) and (2.6), it follows that

$$
\begin{aligned}
\Omega^{1 / 2} \widehat{\nabla}_{a}\left(\Omega^{-1 / 2} \tilde{\psi}\right) & =\Omega^{2}\left[\frac{1}{2} \mathcal{E}_{a b} \tilde{\gamma}^{b}+\frac{1}{12} \alpha \beta \tilde{\gamma}_{a}\right] \tilde{\psi} \\
& +\ldots,
\end{aligned}
$$

Here the dots stand for terms which do not contribute to the spinor charges (see also Appendix A). Inserting this formula into the expression (3.4) for the spinor charge yields the following relation between the Hamiltonian charges $\mathcal{H}_{\xi}$ and the spinor charges $\mathcal{Q}_{\xi}$,

$$
\mathcal{H}_{\xi}=\mathcal{Q}_{\xi}-\int_{S_{\infty}^{2}} W(\alpha) t^{a} \xi_{a} \sqrt{\sigma} d^{2} x
$$


Since the spinor charge is positive and since $\mathcal{H}_{\xi}=E+\omega J$, where $J$ is the angular momentum, this gives

$$
E+\omega J \geq \int_{S_{\infty}^{2}} W(\alpha) \sqrt{\sigma} d^{2} x
$$

and consequently

$$
E \geq 4 \pi \inf W+|J|
$$

Thus the energy is bounded from below in designer gravity for all asymptotic conditions (2.7) that are defined by a function $W(\alpha)$ which is bounded from below.

Note also that, unlike $\mathcal{H}_{\xi}$, the spinor charge $\mathcal{Q}_{\xi}$ is not conserved in general. Instead it depends on the choice of the cross section $S_{\infty}^{2}$ at infinity. This follows immediately from the fact that it differs from $\mathcal{H}_{\xi}$ by the term $\int_{S_{\infty}^{2}} W(\alpha)$, which depends on the choice of the cross section, because $\alpha$ is, in general, time dependent.

Finally, we note that for designer gravity theories that have a dual field theory description, the bound (3.11) is obvious from the point of view of the dual field theory. Indeed, it is known that all configurations in the dual CFT with $W=0$ satisfy $E>=|J|$. The change in the energy by the deformation is $\int_{S_{\infty}^{2}}<W(O(x))>\sqrt{\sigma} d^{2} x$. This leads in the large $N$ limit - which corresponds to the supergravity approximation we used - to $\int_{S_{\infty}^{2}} W(<O>) \sqrt{\sigma} d^{2} x$, which clearly leads to (3.11). The above result, which only involved arguments in the gravity theory, can therefore be viewed as a test of the AdS/CFT correspondence.

\section{Ground State}

Since we have established that the energy $E$ is bounded from below if $W$ has a global minimum, the question arises what are the solutions $\Phi_{0}=\left(g_{a b}, \phi\right)$ that minimize $E$. It is clear from (3.11) that if $W$ is everywhere positive, the ground state of our theory with boundary conditions $\beta=W^{\prime}$ remains pure AdS spacetime with $E=0$. On the other hand if $W$ has negative regions the lower bound (3.11) leaves open the possibility of negative energy solutions which, if they exist, would render pure AdS nonlinearly unstable.

For certain $W$, the solitons discussed in [2] provide explicit examples of negative energy solutions. These solitons are regular, static, spherically symmetric solutions 
with line element

$$
d s^{2}=-h(r) e^{-2 \chi(r)} d t^{2}+h^{-1}(r) d r^{2}+r^{2} d \sigma^{2} .
$$

The set of soliton solutions of our theory (2.2) can be labeled by the value of $\phi$ at the origin. For each $\phi(0)$ and with regular boundary conditions at the origin, one can (numerically) integrate the field equations outward and get a soliton. Asymptotically, $\phi$ behaves like (2.6), so one obtains a point in the $(\alpha, \beta)$ plane. Repeating for all $\phi(0)$ yields a curve $\beta_{s}(\alpha)$ where the subscript indicates this is associated with solitons. We plot this curve ${ }^{6}$ in Fig.1.

For a given $W(\alpha)$, the allowed solitons are simply given by the points where the soliton curve intersects the boundary condition curve: $\beta_{s}(\alpha)=W^{\prime}(\alpha)$. For example, with boundary conditions $\beta(\alpha)=-\alpha^{2}+.22 \alpha^{3}$, which were discussed in [5], one finds precisely one soliton with negative energy, $E_{s} \approx-20 \pi$.

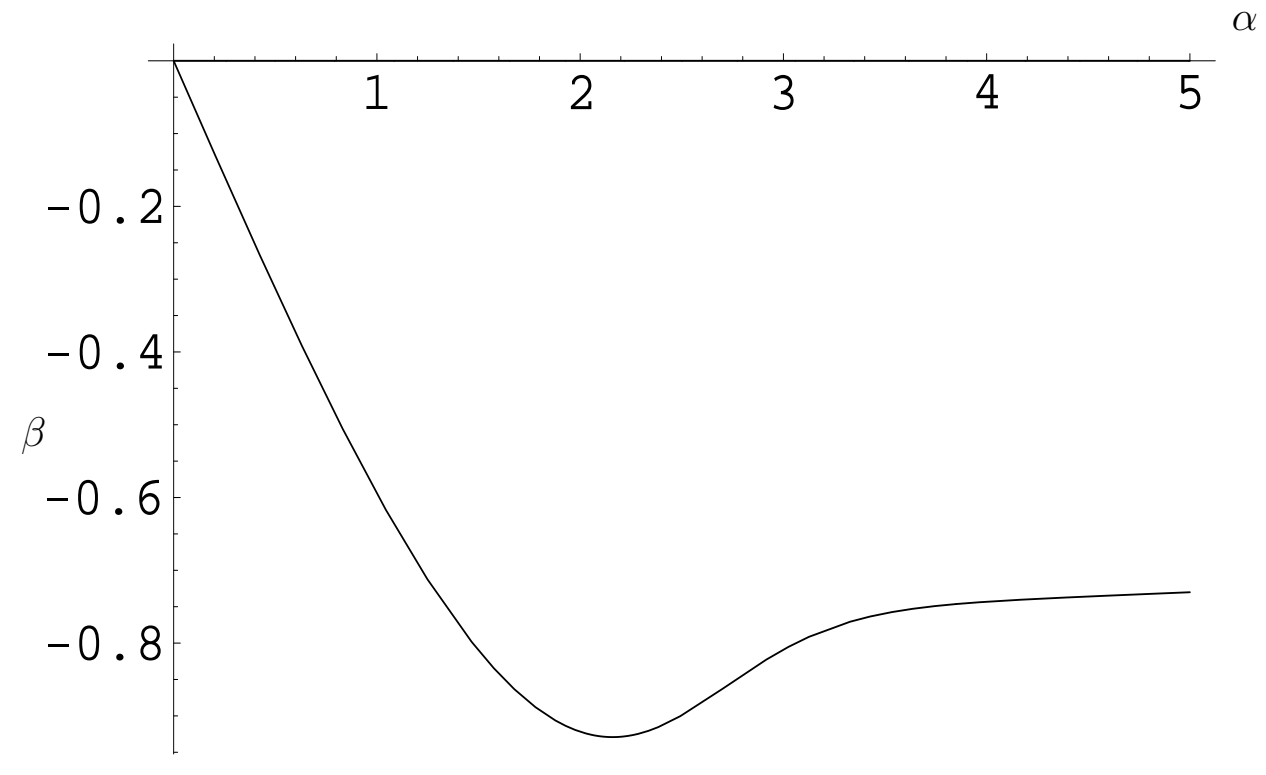

Figure 1: The function $\beta_{s}$ obtained from the solitons.

The AdS/CFT correspondence [6] relates the solitons to nontrivial vacua in the dual field theory, which lives on the boundary $\mathcal{I}$. With $W=0$ boundary conditions,

\footnotetext{
${ }^{6}$ Since $V(\phi)$ is even, it suffices to consider positive $\phi(0)$ corresponding to positive $\alpha$.
} 
the field theory that is dual to $D=4, \mathcal{N}=8$ gauged supergravity is the $2+1$ conformal field theory (CFT) describing the low energy excitations of a stack of M2branes. The scalar $\phi$ that we have retained corresponds to a dimension one operator $\mathcal{O}$ [25]. Imposing more general boundary conditions (2.6) corresponds to the addition of a potential term $\int W(\mathcal{O})$ to the CFT action [7]. According to the standard AdS/CFT dictionary, the solitons determine the expectation value $\langle\mathcal{O}\rangle$ in different vacua of the deformed dual field theory. In particular, given a soliton with $\beta_{s}=W^{\prime}$, one has $\langle\mathcal{O}\rangle=\alpha_{s}$. Furthermore, from the soliton curve $\beta_{s}(\alpha)$ one can construct the following function,

$$
\mathcal{V}(\alpha)=-\int_{0}^{\alpha} \beta_{s}(\tilde{\alpha}) d \tilde{\alpha}+W(\alpha)
$$

which captures this correspondence between solitons and field theory vacua in a nice way. Indeed it can be shown that for any $W$ the location of the extrema of $\mathcal{V}$ yield the vacuum expectation values $\langle\mathcal{O}\rangle=\alpha$, and that the value of $\mathcal{V}$ at each extremum yields the energy of the corresponding soliton [2].

The dual AdS/CFT interpretation of the solitons naturally leads one to conjecture [2] that if $\mathcal{V}$ has a global minimum, the true ground state of the theory with $\beta=W^{\prime}$ boundary conditions should be either the lowest energy soliton (if $E_{s}<0$ ) or pure AdS. In the previous section we have shown that the conserved energy is indeed bounded from below if $W$ (and therefore $\mathcal{V}$ ) has a global minimum. However - although the dual field theory argument is suggestive - this does not immediately imply that the lowest energy state is one of the solitons. This is especially so because the lowest energy soliton does not saturate the lower bound (3.11). Instead the actual soliton energy contains an additional strictly positive contribution, which equals the spinor charge. More generally, it follows from Fig.1 there are $W$ with a negative global minimum (and $W(0)=W^{\prime}(0)=0$ ) which admit no soliton solutions at all. The AdS/CFT interpretation of this is that the corresponding (deformed) field theories have no vacua with $\langle\mathcal{O}\rangle \neq 0$, suggesting pure AdS remains the true ground state of the bulk theory. In fact, our numerical calculations show that unless the global minimum of $W$ is sufficiently negative, all solitons have positive energy (and hence correspond to metastable field theory vacua). These considerations suggest it should be possible to strengthen the bound (3.11).

We have been able to make partial progress towards establishing the nature of the 
ground state in theories where (3.11) provides a nontrivial (negative) lower bound on the energy. If one assumes that the asymptotic value $\alpha_{0}$ of the scalar field in the ground state $\Phi_{0}$ is time-independent ${ }^{7}, \frac{\partial}{\partial t} \alpha_{0}=0$, one can apply a method of Wald and Sudarsky [26] to our theory, to show that $\Phi_{0}$ necessarily has to be static, i.e., that there exists a globally defined time-coordinate $t$ such that the vector field $t^{a}=(\partial / \partial t)^{a}$ Lie-derives the solution, $£_{t} \Phi_{0}=0$ (and therefore is in particular a surface-orthogonal Killing field). We refer the reader to Appendix B for the details of this argument. It essentially consists of proving that if $\Phi_{0}$ is a solution to Einstein's equations, then one can construct a linearized perturbation $\delta \Phi$ satisfying the linearized equations of motion and the linearized boundary conditions, such that $\delta E\left(\Phi_{0}\right)<0$, unless $\Phi_{0}$ is static. A local minimum of the energy by definition has $\delta E\left(\Phi_{0}\right)=0$. Hence it must be static. It remains an open problem, however, to show that the static ground state $\Phi_{0}$ must be spherical, and therefore be the lowest energy soliton. In particular, the fact that we only know the soliton solutions numerically makes it rather difficult to apply the techniques of Masood-ul-Alam [27].

\section{Generalizations}

Although we have concentrated on a single scalar field with $m^{2}=-2$ in four dimensional $\mathcal{N}=8$ supergravity, our arguments apply to more general theories. Consider, for example, gravity in $d$ spacetime dimensions coupled to a scalar field that takes values in an $N$-dimensional Riemannian target space manifold $\left(X, G_{I J}\right)$, with standard kinetic term and potential $V$. Assume that $V$ arises from a superpotential $P$,

$$
V=-8(d-1) P^{2}+8(d-2) G^{I J} P_{, I} P_{, J}
$$

and that $V$ has a critical point $\phi_{0} \in X$ where $V\left(\phi_{0}\right)<0$. This means one can define standard AdS boundary conditions for which the theory admits a positive energy theorem.

However, if some of the scalar masses $m_{I}^{2}$ [the eigenvalues of the matrix $V_{, I}^{J}\left(\phi_{0}\right)$ ] are negative and within the range (2.1), then one can consider alternative asymptotic

\footnotetext{
${ }^{7}$ Numerical calculations provide some support for this claim. We have compared, for several $W$, the energy of solitons to the energy of $O(4)$-invariant instantons, which continue to spherical Lorentzian solutions with $\frac{\partial}{\partial t} \alpha_{0} \neq 0$. In all cases we find the instantons have larger energy than the lowest energy soliton.
} 
conditions that are defined by a smooth function $W: X \rightarrow \mathbb{R}$. As before, one requires there be a conformal factor $\Omega$ such that $\tilde{g}_{a b}=\Omega^{2} g_{a b}$ is smooth at $\mathcal{I}$ and given by eq. (2.5), and one further requires that ${ }^{8}$

$$
\phi^{I}=\phi_{0}^{I}+\Omega^{\lambda_{-}^{I}} \alpha^{I}+\Omega^{\lambda_{+}^{I}} \beta^{I}+\ldots
$$

where $\beta_{I}=W_{, I}$,

$$
\lambda_{ \pm}^{I}=\frac{d-1 \pm \sqrt{(d-1)^{2}+4 m_{I}^{2}}}{2},
$$

and where we have assumed without loss of generality that coordinates in field space have been chosen so that $G_{I J}\left(\phi_{0}\right)=\delta_{I J}$ and $V_{, I J}\left(\phi_{0}\right)=m_{I}^{2} \delta_{I J}$. As in the case of a single scalar field, one can again demonstrate that the consistency condition (2.19) holds, and hence that the generators for the asymptotic symmetries can be consistently defined. The asymptotic symmetry group is generically the isometry group of the $(d-1)$-dimensional Einstein static universe, but for $W=\sum k_{I}\left(\alpha^{I}\right)^{(d-1) / \lambda_{-}^{I}}$ it is the conformal group $O(d-1,2)$. The charges $\mathcal{H}_{\xi}$ must be given by conformally invariant expressions in that case. By analogy with the 4-dimensional case discussed above, we therefore expect that they are given by

$$
\mathcal{H}_{\xi}=-\int_{S_{\infty}^{d-2}} \mathcal{E}_{a b} t^{a} \xi^{b} \sqrt{\sigma} d^{d-2} x-\int_{S_{\infty}^{d-2}}\left[W-\frac{1}{d-1} \sum_{I} \lambda_{-}^{I} \alpha^{I} \beta^{I}\right] t^{a} \xi_{a} \sqrt{\sigma} d^{d-2} x
$$

where $\mathcal{E}_{a b}$ is the leading order electric part of the Weyl tensor given now by

$$
\mathcal{E}_{a b}=\frac{1}{d-3} \Omega^{3-d} \tilde{C}_{a c b d} \tilde{n}^{c} \tilde{n}^{d}
$$

In particular, it would follow that the energy is finite. It may be verified by comparing $\mathcal{H}_{\xi}$ to the spinor charge that it would satisfy a similar lower bound as before, eq. (3.11). A more detailed discussion will appear elsewhere [28].

Finally let us mention that the condition $\beta_{I}=W_{, I}$ is not the most general requirement ensuring that the consistency condition (2.19) is satisfied. In fact, the consistency condition is equivalent to the requirement that the points $\left(\alpha^{I}, \beta_{J}\right) \in \mathbb{R}^{N} \times \mathbb{R}^{N}$ lie on an $N$-dimensional submanifold $S \subset \mathbb{R}^{N} \times \mathbb{R}^{N}$ with the property that the pull-back of the 2-form $d \beta_{I} \wedge d \alpha^{I}$ to $S$ vanishes (such submanifolds are called "Lagrangian").

\footnotetext{
${ }^{8}$ The asymptotic conditions are modified $[3,12]$ for scalar fields which saturate the BF bound, such that $\lambda_{+}=\lambda_{-} \equiv \lambda$.
} 
Locally, one can always find coordinates $\left(\alpha^{I}, \beta_{J}\right)$ and a function $W$ such that $S$ is defined by $\beta_{I}=W_{, I}$, but this need not be the case globally. It would be interesting to carry out our analysis for general Lagrangian submanifolds.

\section{Discussion}

We have studied the stability of designer gravity theories in asymptotically AdS spacetimes, where one couples gravity to a tachyonic scalar (with $m^{2}$ in the range (2.1)) which obeys boundary conditions that are defined by an essentially arbitrary function $W(\alpha)$. We first derived Hamiltonian generators of the asymptotic symmetries in theories of this type. A comparison of the resulting charges with other definitions of charges in asymptotically AdS spaces showed that the generators differ in general from the spinor charges, except when $W=0$. The latter charges are thus not conserved, but their positivity, even in designer gravity, enabled us to establish a lower bound (3.11) on the conserved energy of any solution that satisfies boundary conditions for which $W(\alpha)$ has a global minimum. This proves that a large class of designer gravity theories have a stable ground state.

In cases where $W$ has a negative global minimum, the lowest energy spherical soliton is a natural candidate for the true ground state of the theory. The dual field theory description of certain supergravity theories in particular provides strong evidence for this. It is more difficult, however, to establish the nature of the ground state using purely gravitational arguments. We have only obtained a partial result on this, showing that solutions locally minimizing the energy - and thus in particular the global minimum of the energy - have to be static. One problem is that at present the solitons are only known numerically. A more profound issue is that with boundary conditions that admit negative energy solutions one should not expect the lower bound (3.11) to be saturated by the ground state of the theory. This is because (3.11) does not take in account the contribution from the spinor charge, which is positive and vanishes only in perfect AdS spacetime. It is therefore possible that our bound can be further strengthened, and perhaps even generalized.

Indeed, motivated by the AdS/CFT correspondence, [2] conjectured there would be a lower bound on the energy in all designer gravity theories where the function $\mathcal{V}(\alpha)$, given in eq.(4.2), has a global minimum. It is clear from Fig.1, however, that 
for $\mathcal{V}(\alpha)$ to be bounded from below it is sufficient that $\beta_{s}<W^{\prime}$ at large $\alpha$, which includes certain $W$ that are unbounded ${ }^{9}$. To establish a lower bound on the energy for this class of boundary conditions no doubt requires a better handle on the value of the spinor charge in the true ground state of the theory.

\section{Acknowledgments}

This work was supported in part by NSF grants PHY-0244764 and PHY-0354978 and DOE grant DE-FG02-91ER40618. We would like to thank W. Junker and E. Schrohe, as well as A. Amsel and D. Marolf for discussions.

\section{A Witten Spinor Condition}

We now prove that there exist globally defined, smooth asymptotically Killing spinors satisfying the Witten condition [21]

$$
L \psi \equiv \gamma^{i} \widehat{D}_{i} \psi=0
$$

on a smoothly embedded spacelike 3 -surface $\Sigma \subset M$. We also prove that $\psi$ has a suitable asymptotic expansion near infinity $S_{\infty}^{2}=\partial \Sigma$, and explicitly display the first non-trivial coefficient of that expansion, which is used in the calculations reported in the main text. We assume that $\Sigma$ is so that it can be covered by a finite collection of conical regions, and that its boundary meets $\mathcal{I}$ transversally, implying in particular that the induced metric $h_{i j}$ on $\Sigma$ is asymptotically hyperbolic. We also assume that Einstein's equation hold for $\left(M, g_{a b}, \phi\right)$.

Our strategy for constructing the desired solution to the Witten equation is to first obtain an approximate solution to the Witten equation with the desired asymptotics. This is a smooth spinor field satisfying eq. (A.1) with a source, $L \lambda=J$, where $J$ vanishes to all order in $\Omega$ near $S_{\infty}^{2}$. An exact solution $\psi$ will then be obtained by constructing a suitable solution $\mu$ to the equation $L \mu=-J$, and setting $\psi=\lambda+\mu$.

We seek the approximate solution $\lambda$ as an asymptotic series in $\Omega$,

$$
\lambda \sim \psi_{0}+\Omega \psi_{1}+\Omega^{2} \psi_{2}+\ldots+\Omega^{N} \psi_{N}+\ldots,
$$

\footnotetext{
${ }^{9}$ The dual field theory description suggests that even $W \sim-\alpha^{3}$ boundary conditions yield a well-defined theory [5]. However, the corresponding ground state in the bulk, if it exists, is likely to be a classically singular spacetime.
} 
where $\psi_{0}$ is a Killing spinor in exact AdS-space (i.e., $\widehat{\nabla}_{a} \psi_{0}=0$ in exact AdS with vanishing $\phi$ ) such that asymptotically

$$
\xi^{a}=\bar{\psi}_{0} \gamma^{a} \psi_{0}
$$

for a given (timelike or null) asymptotic symmetry $\xi^{a}$. The spinor fields $\psi_{1}, \psi_{2}, \ldots$ are determined recursively by the condition that $\lambda$ be an asymptotic solution to the Witten equation. The recursion relations are obtained as follows: One first rewrites the Witten equation in terms of the derivative operator associated with the unphysical metric $\tilde{g}_{a b}=\Omega^{2} g_{a b}$, the unphysical gamma matrices $\tilde{\gamma}_{a}=\Omega \gamma_{a}$, and the quantities $\tilde{\psi}_{j}=\Omega^{1 / 2} \psi_{j}$, which are required to be smooth at $S_{\infty}^{2}$. Then the unphysical metric and derivative operator are expanded around the corresponding unphysical metric of exact AdS space as in eq. (2.9). One finally expands the resulting equation in powers of $\Omega$ and equates terms with equal powers. If this is done, then one finds that all spinor fields $\psi_{1}, \psi_{2}, \ldots$ are uniquely determined in terms of $\psi_{0}$. Furthermore, if $\left\{\alpha_{k}\right\}$ is an appropriately fast decreasing sequence of positive real numbers and $\chi$ is a smooth compactly supported function on $\mathbb{R}$ which is equal to 1 in a neighborhood of $\Omega=0$, then the series

$$
\lambda=\sum_{k=0}^{\infty} \chi\left(\Omega / \alpha_{k}\right) \Omega^{k} \psi_{k}
$$

converges, and defines a globally smooth function on $\Sigma$, and $\tilde{\lambda}=\Omega^{1 / 2} \lambda$ is smooth at $S_{\infty}^{2}$. It satisfies the Witten equation with a source $J$ vanishing to all orders in $\Omega$. Clearly, the smooth function $\lambda$ has the asymptotic expansion (A.2), the first terms of which are given explicitly by

$$
\begin{aligned}
\tilde{\psi}_{1}= & 0 \\
\tilde{\psi}_{2}= & \frac{1}{16} \alpha^{2} \tilde{\psi}_{0} \\
\tilde{\psi}_{3}= & -\frac{1}{6} \mathcal{E}_{b c} \tilde{h}_{a}^{b} \tilde{\gamma}^{a} \tilde{\gamma}^{c} \tilde{\psi}_{0}+\frac{1}{36} \alpha \beta \tilde{\psi}_{0} \\
& +\frac{\sqrt{2}-3}{192} \alpha^{2} \tilde{P}_{-} \tilde{U}+\frac{1}{96}\left(\tilde{\gamma}^{d} \tilde{\nabla}_{d} \alpha^{2}-t_{b} \tilde{\gamma}^{b} t^{d} \tilde{\nabla}_{d} \alpha^{2}\right) \tilde{\psi}_{0},
\end{aligned}
$$

where $\tilde{U}$ is a spinor field determined by $\tilde{\psi}_{0}$ that is smooth at scri, and where $\tilde{P}_{ \pm}=$ $\left(1 \pm \tilde{n}^{a} \tilde{\gamma}_{a}\right) / \sqrt{2}$. This expansion is used in obtaining eq. (3.8). 
We next obtain from the asymptotic solution $\lambda$ an exact solution $\psi=\lambda+\mu$ of the Witten equation by constructing a suitable solution $\mu$ to $L \mu=-J$. The existence of such a solution will be established with the help of the following key lemma.

Lemma: Let $\left(M, g_{a b}, \phi\right)$ be a solution of the equations of motion satisfying our asymptotic conditions. Let $u$ be any smooth spinor field on $\Sigma$ of compact support, and let

$$
\|u\|^{2}=\int_{\Sigma}\left[\left(\widehat{D}_{i} u, \widehat{D}^{i} u\right)+\left(1+r^{2}\right)^{-1}|u|^{2}\right] \sqrt{h} d^{3} x,
$$

where $r$ is a radial coordinate equal to $\Omega^{-1}$ near $\partial \Sigma=S_{\infty}^{2}$. Then there exists a positive constant independent of $u$ such that

$$
\text { const. }^{-1}\|u\|^{2} \leq \int_{\Sigma}|L u|^{2} \sqrt{h} d^{3} x \leq \text { const. }\|u\|^{2}
$$

Proof: We begin with the first inequality. We cover $\Sigma$ with a finite number of open cones $\Gamma$, each of which is generated by the orbits of a "radial" vector field $r^{i}$. We normalize this vector field so that $r^{i} D_{i} r=1$. For each such cone, we can define a hermitian, positive definite bilinear form $b(u, v)$ in the spinor bundle, which depends smoothly on the base point, and which is covariantly constant along the integral curves of $r^{i}$ in the sense that

$$
r^{i} D_{i}[b(u, v)]=b\left(r^{i} \widehat{D}_{i} u, v\right)+b\left(u, r^{i} \widehat{D}_{i} v\right) .
$$

Such a hermitian form can be defined e.g. by choosing an arbitrary positive definite hermitian form in the spinor fiber over the apex of $\Gamma$, and then by parallel transporting this form along the integral curves of $r^{i}$ with the connection $r^{i} \widehat{D}_{i}$. From the asymptotic expansion of the metric it is then possible to establish that there is a constant such that

$$
\text { const. }^{-1}\left(1+r^{2}\right)^{-1 / 2} b(u, u) \leq|u|^{2} \leq \text { const. }\left(1+r^{2}\right)^{1 / 2} b(u, u)
$$

at every point in $\Gamma$. Consider now the identity

$$
0=\int_{0}^{\infty} r^{i} D_{i}[r b(u, u)] d r=2 \int_{0}^{\infty} r \operatorname{Re}\left[b\left(u, r^{i} \widehat{D}_{i} u\right)\right] d r+\int_{0}^{\infty} b(u, u) d r .
$$


Integrating this relation over $\widehat{\Gamma}$ (the quotient of $\Gamma$ by its generators) with the surface element $d^{2} \sigma$ of the 2-sphere, using the Cauchy-Schwarz inequality, and using the inequality $r^{2}\left(r^{i} w_{i}\right)^{2} \leq$ const. $h^{i j} w_{i} w_{j}$ for some constant and all $w_{i}$, we obtain

$$
\int_{\Gamma} b(u, u) d r d^{2} \sigma \leq \text { const. } \int_{\Gamma} b\left(\widehat{D}^{i} u, \widehat{D}_{i} u\right) d r d^{2} \sigma .
$$

We now use the geometrical inequality (A.9), together with the fact that the measure $\left(1+r^{2}\right)^{1 / 2} d r d^{2} \sigma$ is equivalent to the measure $\sqrt{h} d^{3} x$ on $\Gamma$. This yields the following inequality,

$$
\int_{\Gamma}\left(1+r^{2}\right)^{-1}|u|^{2} \sqrt{h} d^{3} x \leq \text { const. } \int_{\Gamma}\left(\widehat{D}^{i} u, \widehat{D}_{i} u\right) \sqrt{h} d^{3} x
$$

for some constant. Since $\Sigma$ is the union of finitely many cones $\Gamma$, the above inequality holds also also for the the entire 3-manifold $\Sigma$.

So far we have only used the asymptotic behavior of metric and the scalar field, but not the fact that they satisfy Einstein's equations. We will now use Einstein's equations to derive the first inequality in the lemma from the inequality (A.12). When Einstein's equations are satisfied, then eq. (3.5) holds. Integrating this equation over $\Sigma$ for some compactly supported spinor field $u$ yields

$$
\begin{aligned}
\int_{\Sigma}|L u|^{2} \sqrt{h} d^{3} x & =\int_{\Sigma}\left(\widehat{D}^{i} u, \widehat{D}_{i} u\right) \sqrt{h} d^{3} x \\
& +\frac{1}{2} \int_{\Sigma}\left|\frac{1}{\sqrt{2}} \gamma^{a}\left(\nabla_{a} \phi\right) u+\sinh (\phi / \sqrt{2}) u\right|^{2} \sqrt{h} d^{3} x .
\end{aligned}
$$

Estimating the second term on the right side by 0 from below, and combining the resulting inequality with (A.12) then yields the first half of the inequality in the lemma.

To obtain the second half of the inequality claimed in the lemma, we estimate the second term on the right side instead by

$$
\left|\frac{1}{\sqrt{2}} \gamma^{a}\left(\nabla_{a} \phi\right) u+\sinh (\phi / \sqrt{2}) u\right|^{2} \leq \text { const. }\left(1+r^{2}\right)^{-1}|u|^{2}
$$

from above, which in turn is an immediate consequence of our asymptotic conditions on the metric and the scalar field.//

We now use the lemma to establish a solution to the equation $L \mu=-J$. We first use the lemma to establish the solution of a distributional solution $\mu$. Let $F$ be the 
linear functional on $C_{0}^{\infty}$ defined by

$$
F(u)=-\int_{\Sigma}(J, u) \sqrt{h} d^{3} x
$$

Then, by the Cauchy-Schwartz inequality, and the first half of the inequality in the lemma, we get

$$
\begin{aligned}
|F(u)| & \leq\left(\int_{\Sigma}|u|^{2}\left(1+r^{2}\right)^{-1} \sqrt{h} d^{3} x\right)^{1 / 2}\left(\int_{\Sigma}|J|^{2}\left(1+r^{2}\right) \sqrt{h} d^{3} x\right)^{1 / 2} \\
& \leq \text { const. }\left(\int_{\Sigma}|L u|^{2} \sqrt{h} d^{3} x\right)^{1 / 2} .
\end{aligned}
$$

We interpret this inequality as saying that $F$ is a bounded functional in the scalar product given by the right side of this inequality. Let $H$ be the Hilbert space defined by this inner product (which, by the second inequality of the Lemma is identical with the Hilbert space obtained from $\|\cdot\|)$. By the Riesz representation theorem, there is hence an element $v \in H$ such that $F(u)=\int(L u, L v) \sqrt{h} d^{3} x$ for all $u \in C_{0}^{\infty}$. Again by the inequality in the lemma, every element in $H$ is in particular locally square integrable, so in particular a distribution on $\Sigma$. Thus, $v$ is a solution in the distributional sense of the equation $L^{\dagger} L v=-J$. Hence, $\mu=L^{\dagger} v$ is the desired distributional solution to $L \mu=-J$, and $\psi=\lambda+\mu$ is a global solution to the Witten equation.

It remains to prove that $\psi$ is smooth, and that it has an asymptotic expansion, i.e., can be approximated by ( $\Omega^{-1 / 2}$ times) a polynomial in $\Omega$ with smooth coefficients, up to a function on $\Sigma$ which is smooth everywhere including the boundary, and vanishes to any desired order in $\Omega$. Note that, although this is true by construction for the spinor field $\lambda$, this has not been shown to be the case yet for $\mu$, since it follows from our construction only that $\mu \in L^{2}\left(\Sigma, \sqrt{h} d^{3} x\right)$. However, the machinery developed in [29] will now allow us to establish that $\mu$ is smooth on $\Sigma$ including infinity, and that it has an asymptotic expansion. Following this formalism, we associate with $L$ a "normal operator" $N_{q}(L)$ for each $q \in S_{\infty}^{2}$. This is a differential operator acting on $\left(y^{1}, y^{2}, \Omega\right) \in T_{q} S_{\infty}^{2} \times \mathbb{R}_{+}$, which captures the asymptotics of the operator $L$ near the point $q$. It is given by $N_{q}(L)=\Omega^{3 / 2} N_{+} \Omega^{-1 / 2}$, where

$$
N_{ \pm}=\Gamma \partial_{\Omega}+D_{\mathbb{R}^{2}} \mp \frac{3}{2} \Omega^{-1}(1 \mp \Gamma)
$$


and where $D_{\mathbb{R}^{2}}=\gamma^{1} \partial_{y^{1}}+\gamma^{2} \partial_{y^{2}}$ is the ordinary Dirac operator on $\mathbb{R}^{2}$, and where $\Gamma=i \gamma^{1} \gamma^{2}$. The dependence upon $q$ has dropped out in the present case because $L$ is spherically symmetric asymptotically. One next determines for which $\delta \in \mathbb{R}$ the operator $N_{q}(L)$ has a nontrivial kernel in the space $\Omega^{\delta} L^{2}\left(\Omega^{-3} d \Omega d^{2} y\right)$ (the integration element reflects the large distance behavior of the volume element $\left.\sqrt{h} d^{3} x \sim \Omega^{-3} d \Omega d^{2} \sigma\right)$. This kernel may be determined e.g. by noting that any element in its kernel necessarily has to be $\Omega^{1 / 2}$ times an element in the kernel of $N \equiv N_{-} N_{+}$, which in turn may be identified with the matrix operator

$$
N=\left(\begin{array}{cc}
\partial_{\Omega}^{2}+3 \Omega^{-1} \partial_{\Omega}-|k|^{2} & 0 \\
0 & \partial_{\Omega}^{2}+3 \Omega^{-1} \partial_{\Omega}-|k|^{2}-3 \Omega^{-2}
\end{array}\right),
$$

acting on functions of $\Omega \in \mathbb{R}_{+}$, where we have also performed a Fourier transform in $y^{1}, y^{2}$. The equation $N\left(\chi_{1}, \chi_{2}\right)=0$ can be solved in terms of Bessel functions. It turns out that $\chi_{1}$ must be a linear combination $a_{1} \Omega^{-1} I_{1}(|k| \Omega)+a_{2} \Omega^{-1} K_{1}(|k| \Omega)$, while $\chi_{2}$ must be a linear combination $b_{1} \Omega^{-1} I_{2}(|k| \Omega)+b_{2} \Omega^{-1} K_{2}(|k| \Omega)$. Thus, no element in the kernel of $N_{q}(L)$ can be in $\Omega^{\delta} L^{2}\left(\mathbb{R}_{+}, \Omega^{-3} d \Omega\right)$ for $\delta>-2$, in particular, $N_{q}(L)$ is injective on $L^{2}\left(\mathbb{R}_{+}, \Omega^{-3} d \Omega\right)$. Since $L \mu=-J, \mu \in L^{2}\left(\Omega^{-3} d \Omega d^{2} \sigma\right)$, and since $J$ has an asymptotic expansion near the boundary, it now follows by Prop. 7.17 of [29], that also $\mu$ is smooth and that it has an asymptotic expansion, with leading coefficient $\Omega^{-1 / 2}$. Consequently, also $\psi=\lambda+\mu$ has such an expansion, which is what we desired to show. Actually, since the expansion of $\psi$ is unique (and hence equal to that of $\lambda$ by construction), the expansion of $\mu$ must in fact vanish, i.e., $\mu$ must in fact vanish to all orders in $\Omega$.

\section{B Staticity of Extrema of Energy}

In this Appendix we show that if the asymptotic value $\alpha_{0}$ of the scalar field is timeindependent, i.e. $\frac{\partial}{\partial t} \alpha_{0}=0$, then solutions $\Phi_{0}=\left(g_{a b}, \phi\right)$ that minimize the energy $E=$ $\mathcal{H}_{\partial / \partial t}$ must be static, i.e., there exists a globally defined time-coordinate $t$ such that the vector field $t^{a}=(\partial / \partial t)^{a}$ Lie-derives the solution, $£_{\partial / \partial t} \Phi_{0}=0$ (and therefore is in particular a surface-orthogonal Killing field). We prove this by applying a method of Wald and Sudarsky [26] to our theory. This method proceeds by showing that, if $\Phi_{0}$ is a solution to Einstein's equation, then one can construct a linearized perturbation $\delta \Phi$ satisfying the linearized equations of motion and the linearized boundary conditions, 
such that $\delta E\left(\Phi_{0}\right)<0$, unless $\Phi_{0}$ is static. A local minimum of the energy by definition has $\delta E\left(\Phi_{0}\right)=0$. Hence it must be static.

In order to construct the desired perturbation, we make the additional technical assumption that our energy minimizing asymptotically AdS solution $\Phi_{0}=\left(\phi, g_{a b}\right)$ is such that the metric admits a foliation by spacelike maximal 3-surfaces $\Sigma$ (i.e., surfaces with vanishing trace $K$ of the extrinsic curvature), meeting infinity $\mathcal{I}$ transversally. The desired perturbation can be constructed as follows. Pick a maximal surface $\Sigma$, let $h_{i j}$ and $K_{i j}$ be the induced metric and extrinsic curvature, and let $\phi$ and $p$ be the scalar field and its normal derivative on $\Sigma$. Assuming that our theory has a well-posed initial value formulation, these can be identified with initial data for the solution $\Phi_{0}$. We now consider initial data for the linearized solution $\delta \Phi$ of the form

$$
\delta K_{i j}=-6 f K_{i j}-K_{i j}, \delta h_{i j}=4 f h_{i j}, \delta p=-6 f p-p, \delta \phi=0,
$$

where $f$ is a function on $\Sigma$ to be determined. Equations (B.1) correspond to a linearized solution on $M$ if and only if $f$ is chosen such that the linearized constraint equations $\delta \mathbf{C}_{a}=0$ are satisfied. Under our assumption $K=0$, these are equivalent to the equation

$$
D_{i} D^{i} f-\mu f=\rho,
$$

where

$$
\mu=K_{i j} K^{i j}+p^{2}+2+\cosh (\sqrt{2} \phi), \quad \rho=\frac{1}{4} K_{i j} K^{i j}+\frac{1}{4} p^{2} .
$$

Equation (B.2) is a Laplace equation for the asymptotically hyperbolic metric $h_{i j}$, with a potential and a source. It follows from $\frac{\partial}{\partial t} \alpha_{0}=0$ that the potential satisfies $\mu=3+O\left(r^{-2}\right)$ and that the source satisfies $\rho=O\left(r^{-6}\right)$. One can establish using the methods of $[29,30]$ that such an equation (B.2) has an everywhere smooth solution $f$ which asymptotically behaves like $r^{-3} f_{0}$, with $f_{0}$ a smooth function on $\partial \Sigma=S_{\infty}^{2}$, the cut at infinity. In particular, the corresponding linearized perturbation $\delta \Phi$ will then satisfy the linearized asymptotic conditions. In order to calculate the change in the energy associated with this linearized perturbation, we note that due to the rapid fall-off of $f$ at infinity, $\delta \Phi$ has a vanishing $\delta \alpha$. Hence, the change in energy for the linearized perturbation comes entirely from the Weyl-tensor term in eq. (2.22), and is given by

$$
\delta E\left(\Phi_{0}\right)=-\delta \int_{S_{\infty}^{2}} \mathcal{E}_{a b} t^{a} t^{b} \sqrt{\sigma} d^{2} x
$$




$$
\begin{aligned}
= & -\lim _{r \rightarrow \infty} \delta \int_{S_{r}^{2}} r^{5}\left(\mathcal{R}_{i j}-K_{i k} K_{j}^{k}-\frac{1}{3} p^{2} h_{i j}\right. \\
& \left.\quad-\frac{1}{6} h_{i j} D^{k} \phi D_{k} \phi-\frac{1}{3} h_{i j} V-\frac{1}{2} D_{i} \phi D_{j} \phi\right) r^{i} r^{j} \sqrt{\sigma} d^{2} x \\
= & 24 \int_{S_{\infty}^{2}} f_{0} \sqrt{\sigma} d^{2} x,
\end{aligned}
$$

where in the second line, we have used the Gauss-Codacci equations and Einstein's equation, and where $\mathcal{R}_{i j}$ is the Ricci tensor of $h_{i j}$. Because $\rho, \mu \geq 0$, the elliptic equation (B.2) is of a form to which the Hopf maximum principle [31] applies, that is, within each domain $D \subset \Sigma$ the solution $f$ must assume its maximum on $\partial D$, or it must be constant. However, $f$ vanishes at infinity, so $f \leq 0$ everywhere. Consequently, also $f_{0} \leq 0$, meaning that $\delta E\left(\Phi_{0}\right) \leq 0$. Moreover, we will now argue that, if $\rho \neq 0$, then also $f_{0} \neq 0$, and so we must have in fact that $\delta E\left(\Phi_{0}\right)<0$, meaning that the perturbation causes the energy to decrease (to first order). Consequently, in order to avoid a contradiction with $\delta E\left(\Phi_{0}\right)=0$ for minimizing solutions, it must be true that $\rho=0$ and therefore that

$$
p=0, \quad K_{i j}=0
$$

everywhere on the maximal 3-surface $\Sigma$. Since $M$ can be foliated by such surfaces, this implies that the solution $\Phi_{0}$ is globally static. Thus, in order to finish the proof, we must show that $f_{0}=0$ implies $\rho=0$. The proof of this is accomplished by the following lemma.

Lemma: Let $f$ be a smooth solution to $D^{i} D_{i} f-\mu f=\rho$, where $\mu$ and $\rho$ are smooth, nonnegative functions on $\Sigma$ satisfying $\mu=3+O\left(r^{-2}\right)$ and $\rho=O\left(r^{-2}\right)$, and where $D_{i}$ is the derivative operator of an asymptotically hyperbolic metric $h_{i j}$. Assume that $f=O\left(r^{-3-\epsilon}\right)$ for some $\epsilon>0$. Then $\rho=0=f$.

Proof: We can write the asymptotically hyperbolic metric on $\Sigma$ as

$$
h_{i j} d x^{i} d x^{j}=\Omega^{-2}\left(d \Omega^{2}+k^{2} d \sigma^{2}\right)
$$

where $\Omega=O\left(r^{-1}\right)$ is a non-negative defining function of the surface $S_{\infty}^{2}$ at infinity, and $k$ is a smooth function of $\Omega$ and the angles such that $k \rightarrow 1$ and $k^{\prime} \rightarrow 0$ as $\Omega \rightarrow 0$. 
Prime denotes a derivative with respect to $\Omega$. Consider now the quantity

$$
G(\Omega)=-\Omega^{-1} \int_{S_{\Omega}^{2}} k^{-2} f \sqrt{\sigma} d^{2} x .
$$

By the maximum principle, we have $G \geq 0$, and from the assumption about the asymptotics of $f$, we know that $\Omega^{-2-\epsilon} G(\Omega)$ is continuous as $\Omega \rightarrow 0$, for some $\epsilon>0$. We will now show that there must be an $\Omega>0$ for which $G$ vanishes. This will finish the proof, for then we must have that $f(x)=0$ at some interior point $x \in \Sigma$. Indeed, the Hopf maximum principle leaves only the alternatives that the global maximum of $f$ (equal to 0 ) must be attained on the boundary $\partial \Sigma$, or that $f$ vanishes identically. Thus, the second alternative must be the case, implying that also $\rho=0$ identically.

To prove that $G=0$ for some $\Omega>0$, we integrate the equation for $f$ over $S_{\Omega}^{2}$, and use that $f \leq 0, \rho \geq 0$ one obtains an evolution equation for $G$ of the form

$$
\left(\Omega^{-1}(\Omega G)^{\prime}\right)^{\prime} \leq 3 \Omega^{-2} G+(\Omega H)^{\prime}+F,
$$

where

$$
\begin{aligned}
& H(\Omega)=-2 \Omega^{-2} \int_{S_{\Omega}^{2}} k^{-2}(\log k)^{\prime} f \sqrt{\sigma} d^{2} x \\
& F(\Omega)=-\Omega^{-1} \int_{S_{\Omega}^{2}} k^{-2} \xi f \sqrt{\sigma} d^{2} x .
\end{aligned}
$$

where $\xi=\Omega^{-2}\left[p^{2}+K_{i j} K^{i j}+2 \sinh ^{2}(\phi / \sqrt{2})\right] \geq 0$ is smooth as $\Omega \rightarrow 0$. We now integrate the inequality from 0 to $\Omega$, using the estimate

$$
\int_{0}^{\Omega} x^{-2} G(x) d x \leq\left(\sup _{0 \leq x \leq \Omega} x^{-2-\epsilon} G(x)\right) \int_{0}^{\Omega} x^{\epsilon} d x=\frac{1}{1+\epsilon} \Omega^{1+\epsilon} \gamma(\Omega)
$$

for the first term, where we defined

$$
\gamma(\Omega)=\sup _{0 \leq x \leq \Omega} x^{-2-\epsilon} G(x) .
$$

We furthermore use the estimate

$$
\Omega H(\Omega) \leq\left(\sup _{0 \leq x \leq \Omega} x^{-1}\left|(\log k)^{\prime}(x)\right|\right) \Omega^{3+\epsilon} \gamma(\Omega) \equiv \Omega^{3+\epsilon} c_{1}(\Omega) \gamma(\Omega)
$$


for the second term, and the estimate

$$
\begin{aligned}
\int_{0}^{\Omega} F(x) d x & \leq\left(\sup _{0 \leq x \leq \Omega} \xi(x)\right)\left(\sup _{0 \leq x \leq \Omega} x^{-2-\epsilon} G(x)\right) \int_{0}^{\Omega} x^{1+\epsilon} d x \\
& \equiv \Omega^{2+\epsilon} c_{2}(\Omega) \gamma(\Omega)
\end{aligned}
$$

for the third term on the right side. Note that all three functions $c_{1}, c_{2}, \gamma$ are nonnegative, continuous at $\Omega=0$, and monotonically increasing. From these estimates, we get the inequality

$$
(\Omega G)^{\prime} \leq \frac{3}{1+\epsilon} \Omega^{2+\epsilon} \gamma(\Omega)+c_{1}(\Omega) \Omega^{3+\epsilon} \gamma(\Omega)+c_{2}(\Omega) \Omega^{4+\epsilon} \gamma(\Omega)
$$

Now pick a fixed $\Omega_{0}>0$, and integrate one more time from 0 to $\Omega \leq \Omega_{0}$. Then one obtains the inequality

$$
\Omega^{-2-\epsilon} G(\Omega) \leq\left(\frac{3}{(1+\epsilon)(3+\epsilon)}+\frac{1}{4+\epsilon} c_{1}\left(\Omega_{0}\right) \Omega_{0}+\frac{1}{5+\epsilon} c_{2}\left(\Omega_{0}\right) \Omega_{0}^{2}\right) \gamma\left(\Omega_{0}\right) .
$$

maximizing the left side over all $\Omega \leq \Omega_{0}$ then gives the final inequality

$$
\gamma\left(\Omega_{0}\right) \leq\left(\frac{3}{(1+\epsilon)(3+\epsilon)}+\frac{1}{4+\epsilon} c_{1}\left(\Omega_{0}\right) \Omega_{0}+\frac{1}{5+\epsilon} c_{2}\left(\Omega_{0}\right) \Omega_{0}^{2}\right) \gamma\left(\Omega_{0}\right) .
$$

It is now clear that if $\Omega_{0}$ is chosen small enough, then the first term on the right side will dominate the other terms, and we get an inequality of the form $\gamma\left(\Omega_{0}\right) \leq$ $(1-\delta) \gamma\left(\Omega_{0}\right)$, where $\delta>0$. This is possible only if in fact $\gamma\left(\Omega_{0}\right)=0$ and hence $G\left(\Omega_{0}\right)=0$, which is what we wanted to show.//

\section{References}

[1] P. Breitenlohner and D. Z. Freedman, "Stability In Gauged Extended Supergravity," Annals Phys. 144 (1982) 249; "Positive Energy In Anti-De Sitter Backgrounds And Gauged Extended Supergravity," Phys. Lett. B 115 (1982) 197

[2] T. Hertog, G. T. Horowitz, "Designer Gravity and Field Theory Effective Potentials," Phys. Rev. Lett. 94 (2005) 221301, hep-th/0412169

[3] T. Hertog, K. Maeda, "Black Holes with Scalar Hair and Asymptotics in $N=8$ Supergravity," JHEP 0407 (2004) 051, hep-th/0404261 
[4] C. Martinez, R. Troncoso, J. Zanelli, "Exact Black Hole Solution with a Minimally Coupled Scalar Field," Phys. Rev. D 70 (2004) 084035, hep-th/0406111

[5] T. Hertog, G. T. Horowitz, "Holographic Description of AdS Cosmologies," JHEP 0504 (2005) 005, hep-th/0503071

[6] J. M. Maldacena, "The large N limit of superconformal field theories and supergravity," Adv. Theor. Math. Phys. 2 (1998) 231, hep-th/9711200

[7] E. Witten, "Multi-Trace Operators, Boundary Conditions, and AdS/CFT Correspondence," hep-th/0112258

[8] M. Berkooz, A. Sever and A. Shomer, "Double-trace deformations, boundary conditions and spacetime singularities," JHEP 0205 (2002) 034, hep-th/0112264; A. Sever and A. Shomer, "A note on multi-trace deformations and AdS/CFT," JHEP 0207 (2002) 027, hep-th/0203168.

[9] R. M. Wald, General Relativity, The University of Chicago Press, Chicago 1984

[10] M. J. Duff, J. T. Liu, "Anti-de Sitter Black Holes in Gauged N=8 Supergravity," Nucl. Phys. B554 (1999) 237, hep-th/9901149

[11] S. Hollands, A. Ishibashi, D. Marolf, "Comparison Between Various Notions of Conserved Charges in Asymptotically AdS-Spacetimes," hep-th/0503045

[12] M. Henneaux, C. Martinez, R. Troncoso, J. Zanelli, "Asymptotically Anti-de Sitter Spacetimes and Scalar Fields with a Logarithmic Branch," Phys. Rev. D 70 (2004) 044034, hep-th/0404236

[13] M. Henneaux, C. Teitelboim, "Asymptotically Anti-de Sitter Spaces," Comm. Math. Phys. 98 (1985) 391.

[14] R. M. Wald, A. Zoupas, "A General Definition of Conserved Quantities in General Relativity and Other Theories of Gravity," Phys. Rev. D61 (2000) 084027, hep-th/9911095

[15] A. Ashtekar, A. Magnon, "Asymptotically Anti-de Sitter Spacetimes," Class. Quant. Grav. Lett. 1 (1984) L39 
[16] M. Henningson and K. Skenderis, "The holographic Weyl anomaly," JHEP 9807, 023 (1998) [arXiv:hep-th/9806087];

[17] V. Balasubramanian and P. Kraus, "A stress tensor for anti-de Sitter gravity," Commun. Math. Phys. 208, 413 (1999) [arXiv:hep-th/9902121].

[18] S. Hollands, A. Ishibashi and D. Marolf, "Counter-term charges generate bulk symmetries," arXiv:hep-th/0503105.

[19] T. Hertog, G. T. Horowitz, "Towards a Big Crunch Dual," JHEP 0407 (2004) 073, hep-th/0406134

[20] I.R. Klebanov, E. Witten, "AdS/CFT Correspondence and Symmetry Breaking," Nucl. Phys. B556 (1999) 89, hep-th/9905104

[21] E. Witten, "A Simple Proof Of The Positive Energy Theorem," Commun. Math. Phys. 80 (1981) 381.

[22] L. F. Abbott, S. Deser, "Stability of Gravity with a Cosmological Constant," Nucl. Phys. B195 (1982) 76

[23] G. W. Gibbons, C. M. Hull and N. P. Warner, "The Stability Of Gauged Supergravity," Nucl. Phys. B 218 (1983) 173

[24] P.K. Townsend: "Positive Energy and the Scalar Potential in Higher Dimensional (Super) Gravity Theories," Phys. Lett. B 148 (1984) 55

[25] O. Aharony, Y. Oz and Z. Yin, "M-theory on $\operatorname{AdS}(\mathrm{p}) \times \mathrm{S}(11-\mathrm{p})$ and superconformal field theories," Phys. Lett. B 430 (1998) 87, hep-th/9803051

[26] D. Sudarsky and R. M. Wald, "Extrema of mass, stationarity, and staticity, and solutions to the Einstein Yang-Mills equations," Phys. Rev. D 46 (1992) 1453.

[27] A. K. M. Masood-ul-Alam, "Uniqueness of a Static Charged Dilaton Black Hole," Class. Quant. Grav. 10 (1993) 2649

[28] A. Amsel, to appear 
[29] R. Mazzeo: "Elliptic theory of differential edge operators I," Commun. Part. Diff. Equations, 16 (10) (1991), 1615-1664

[30] R. Mazzeo: "The Hodge Cohomology of a conformally compact metric," J. Diff. Geom. 28 (1988) 309-339

[31] See e.g. R. Courant and D. Hilbert: Methods in Mathematical Physics, Interscience, New York 1962, Vol. 2 